\title{
Association of the polymorphisms of TRAF1 (rs10818488) and TNFAIP3 (rs2230926) with rheumatoid arthritis and systemic lupus erythematosus and their relationship to disease activity among Egyptian patients
}

\author{
MAI MOAAZI, NEVINE MOHANNAD \\ 'Department of Immunology and Allergy, Medical Research Institute, Alexandria University, Alexandria, Egypt \\ ${ }^{2}$ Department of Internal Medicine Rheumatology Unit, Alexandria University Hospitals, Alexandria, Egypt
}

\begin{abstract}
Aim of the study: Recent studies demonstrated the association of tumor necrosis factor $\alpha$-induced protein 3 (TNFAIP3) (rs2230926) and tumor necrosis factor receptor associated factor 1 (TRAF1) (rs10818488) with rheumatoid arthritis (RA) and systemic lupus erythematosus (SLE) in different populations. We aimed at determining whether they confer susceptibility to SLE and RA in Egyptian population and if there is any relation to disease activity and auto-antibodies profile.

Material and methods: A case-control study involving 105 individuals with RA, 90 with SLE and 75 healthy controls was performed using TaqMan genotyping assay for two SNPs that showed the best evidence of association in the previous Caucasian studies.

Results: We detected significant differences in $G$ allele frequency of TNFAIP3 (rs2230926) with $\operatorname{SLE}\left(p=0.017^{*}\right)$ and $R A\left(O R=2.333 ; 95 \% C I: 1.103-4.935, p=0.023^{*}\right)$ and association with RA disease activity $(<0.001)$. The A allele of TRAF1 was significantly increased in RA compared to controls $(p=0.049)$ and with RA activity $(p=0.001)$, while TRAF1 polymorphism did not exhibit any significant difference in the frequencies of genotypes or alleles in SLE and control $(p=0.280)$.

Conclusions: TNFAIP3 is a susceptibility gene to SLE and RA in the Egyptian population and is correlated to disease activity and the presence of autoantibodies while TRAF1 polymorphisms increase the risk of RA but not to SLE in Egyptian populations.
\end{abstract}

Key words: rheumatoid arthritis, systemic lupus erythematosus, TNFAIP3 gene, TRAF1.

(Cent Eur J Immunol 2016; 41 (2): 165-175)

\section{Introduction}

Rheumatoid arthritis (RA) and systemic lupus erythematosus (SLE) are both the most heterogeneous complex autoimmune diseases. RA is a systemic, chronic inflammatory disorder that affects different tissues and organs. It mainly attacks the synovial joints leading to an inflammatory response in the synovium (synovitis) and hyperplasia of the synovial cells as well as excess synovial fluid [1]. Disease onset is most frequent during the fourth and fifth decades of life, with $80 \%$ of patients developing the disease between the ages of 35 and 50. The incidence of RA is six times greater in females aged 60-64 years compared to 18-29-year-old females. Its prevalence rises with age, and sex variances are reduced in the older age group. RA is present all over the world and affects all races. Yet, both incidence and severity appear to be lower in rural sub-Saharan Africa and in Caribbean blacks [2].

Systemic lupus erythematosus is a systemic disease characterized by the production of autoantibodies against various nuclear antigens owing to a breakdown in self-tolerance that can affect several organs or organ systems, resulting in a broad spectrum of clinical and immunologic manifestations [3]. Prevalence of SLE in the population is 20 to 150 cases per 100,000 and it may have a genetic link [2].

Although the etiologies of RA and SLE remain unclear, it is widely accepted that each of these two autoimmune diseases is multifactorial and caused by an interaction of a variety of genetic and environmental factors [4]. Recent-

Correspondence: Prof. Mai Moaaz, Department of Immunology and Allergy, Medical Research Institute, Alexandria University, Alexandria, Egypt, e-mail: mai_moaaz@yahoo.com

Submitted: 25.01.2016, Accepted: 10.02.2016 
ly, genome-wide association studies (GWAS) have led to the association of a number of new susceptibility genes with SLE and RA [5, 6].

Tumor necrosis factor $\alpha$-induced protein 3 (TNFAIP3) gene encodes the ubiquitin-modifying enzyme; A20. Reduced negative regulatory activity of A20 protein may lead to unnecessary immune activity, causing enhanced autoreactivity [7]. SNP rs2230926, a nonsynonymous mutation (c.380T $>\mathrm{G})$, encoding a phenylalanine or cysteine at amino acid position 127 of the protein, is located in exon 3 of TNFAIP3, which might affect the function of TNFAIP3 gene related to SLE and RA [8].

Moreover, tumor necrosis factor receptor-associated factor-1 (TRAF1) gene encodes an intracellular protein member of the TNF receptor-associated factor family involved in TNF- $\alpha$ signaling [9], which is considered to be associated with the activation and proliferation of $\mathrm{T}$ cells [10]. Therefore, TRAF1 might possibly contribute to the pathogenesis of both autoimmune diseases; RA and SLE. Association between single nucleotide polymorphism (SNP) of TRAF1 polymorphism rs10818488 and SLE and RA has been reported in different ethnic groups [3, 11, 12]. Typically, Lee et al. [13] reported that the allelic frequencies of genes often differ substantially in different ethnic groups, and thus, ethnically specific association studies are required to determine genetic associations in different populations.

This is the first study to attempt to determine the potential implication of the TNFAIP3 polymorphism and replicated study of the TRAF1 polymorphism with RA in an Egyptian population. We attempted to validate the obscure associations of these two SNPs with the susceptibility to SLE and RA in Egyptian population and their association with disease activity and the presence of autoantibodies.

\section{Material and methods}

\section{Subjects}

All patients were recruited from the Rheumatology unit in the Alexandria Main University hospital in the period from April 2013 to July 2014. The subjects in the RA group consisted of 105 Egyptian patients with chronic RA. All patients with RA fulfilled the 2010 ACR/EULAR (American College of Rheumatology/European League Against Rheumatism) criteria for RA [14]. RA patients were classified into groups according to disease activity as reflected by DAS28 scoring system [15]. Predefined cut offs for remission, mild, moderate and severe disease activity according to EULAR criteria are < 2.6, 2.6-3.2, $>3.2-<5.1$ and $>5.1$, respectively [15].

The subjects in the SLE study group consisted of 90 Egyptian patients with chronic SLE. All SLE patients fulfilled the 2012 revised SLICC criteria for classification of SLE [16]. All the patients had documented medical histo- ries of SLE and were subjected to a full history-taking and thorough clinical examination. In this study, the overall disease activity was scored using SLE Disease Activity Index (SLEDAI) to assess lupus activity [17]. SLE patients were arbitrarily categorized into 2 groups: mild to moderate flare (SLEDAI score > 3) and severe flare (SLEDAI > 12).

The control group was frequency matched to cases by age interval and included 75 subjects. Exclusion criteria for controls included prior personal history of any autoimmune diseases. Patients and controls were chosen as free from infectious or other autoimmune diseases that may interfere with results of the study. All subjects gave informed consent approved by local ethics committee. Demographic data were obtained via a standardized questionnaire. There were no reports of genotoxic medications, alcohol consumption, or exposure to radiation for at least one month before.

\section{Methods}

\section{Immunological analysis}

Peripheral blood samples were collected from all cases and matched healthy controls. Sera were separated immediately and stored at $-20^{\circ} \mathrm{C}$. C-reactive protein (CRP) was measured using high-sensitivity enzyme-linked immunosorbent assay (ELISA) [18]. For RA patients, IgM rheumatoid factor (RF) was measured by latex agglutination [19]. RF was considered positive if above $20 \mathrm{IU} / \mathrm{ml}$. Anti-CCP was detected in serum samples using enzyme-linked immunosorbent assay test. The assay was performed according to the manufacturer's instructions [20]. A concentration $>25 \mathrm{IU} / \mathrm{ml}$ was considered positive. For SLE subjects, ANA was detected by indirect immuno-fluorescence. Antidouble stranded DNA (dsDNA) antibody (Anti-ds DNA) was detected in serum samples using enzyme-linked immunosorbent assay test. A concentration $>30 \mathrm{IU} / \mathrm{ml}$ was considered positive.

\section{DNA isolation from PBMCS}

DNA was isolated and purified from peripheral blood mononuclear cells (PBMCs) isolated via density gradient centrifugation with Ficoll hypaque (1077) solution (Biochrom KG Berlin) using QIAamp-spin-columns according to the protocol provided by the manufacturer (QIAamp Blood Kit; Qiagen GmbH, Hilden, Germany) [21].

\section{Analysis of rs2230926 (TNFAIP3) and rs10818488 (TRAF1) polymorphism}

Genotyping of SNP was carried out using the TaqMan genotyping assay (Applied Biosystems-Life Technologies, Carlsbad, California, USA), according to the manufacturer's instructions [22]. Predesigned TaqMan SNP genotyping assays were used (probe ID: rs2230926, C_770116_10) and (probe ID: rs10818488, C_2783655_10). Amplification was performed in a $10 \mu \mathrm{l}$ reaction volume containing $5 \mu \mathrm{l}$ of TaqMan genotyping Master Mix and $0.125 \mu \mathrm{l}$ of TaqMan 
genotyping assay mix using the Step One real-time PCR system (Applied Biosystems-Life Technologies, Carlsbad, California, USA). Thermal cycling conditions consisted of initial denaturation at $95^{\circ} \mathrm{C}$ for 10 minutes, followed by 40 cycles of $95^{\circ} \mathrm{C}$ for 15 seconds and $60^{\circ} \mathrm{C}$ for 1 minute.

The resultant PCR products were resolved by electrophoresis on $3 \%$ agarose gel, and stained with ethidium bromide for visualization under UV light.

\section{Statistical methods}

Statistical analyses were performed by SPSS.18. Descriptive measures were done for all variables and a $p$-value less than 0.05 was considered statistically significant. Student's $t$ test, chi-squared $\left(\chi^{2}\right)$ test and Fisher exact test were used to assess the general characteristics of groups.

Agreement with Hardy-Weinberg equilibrium (HWE) was assessed by comparing expected to observed genotype frequencies. The odds ratios (ORs) and $95 \%$ confidence intervals (95\% CIs) from unconditional logistic regression were measured. The conditional logistic regression model was used to estimate adjusted ORs according to potential confounders selected a priori. The population-attributable risk was calculated using the formula: $\mathrm{P}_{\mathrm{Ho}}\left(\mathrm{OR}_{\mathrm{Ho}}-1\right)+\mathrm{P}_{\mathrm{He}}\left(\mathrm{OR}_{\mathrm{He}}-1\right) /\left(1+\mathrm{P}_{\mathrm{Ho}}\left(\mathrm{OR}_{\mathrm{Ho}}-1\right)+\mathrm{P}_{\mathrm{He}}\right.$ $\left(\mathrm{OR}_{\mathrm{He}}-1\right)$, where $\mathrm{P}_{\mathrm{Ho}}$ and $\mathrm{P}_{\mathrm{He}}$ are the proportions of homozygotes and heterozygotes of the risk allele, respectively, while $\mathrm{OR}_{\mathrm{Ho}}$ and $\mathrm{OR}_{\mathrm{He}}$ are the estimated ORs for homozygotes and heterozygotes of the risk allele, respectively. Because one polymorphism was being investigated for each gene, significance test results were quoted as two-tailed probabilities. Significance of the obtained results was judged at the $5 \%$ level.

\section{Results}

\section{Hardy-Weinberg equilibrium}

Genotype distributions at each studied locus were consistent with HWE in cases and controls (Table 1).

\section{Clinical characteristics of RA patients}

The RA patients included 27 (25.7\%) males and 78 $(74.3 \%)$ females, controls included $25(33.3 \%)$ males and $50(66.7 \%)$ females $(p=0.266)$ with mean age of 38.95 \pm 9.04 . Mean \pm SD of RA patient's age was $41.91 \pm 10.63$ years. The mean \pm SD of disease duration was $4.59 \pm 4.58$ years, the mean \pm SD of ESR was $56.31 \pm 33.08 \mathrm{~mm} / \mathrm{hr}$ while that of controls was $9.40 \pm 2.01(p<0.001)$, and the mean \pm SD of CRP concentration was $24.73 \pm 18.99 \mathrm{mg} / \mathrm{l}$ and that of controls was $2.19 \pm 0.44(p<0.001)$. Moreover, $42(40.0 \%)$ patients were RF positive, and 80 (76.2\%) were anti-CCP positive (Table 2).

\section{Association of TNFAIP3 (rs2230926) and TRAF1 (rs10818488) polymorphism genotypes with RA susceptibility}

Our results revealed a significant association between TNFAIP3 rs2230926 and RA when comparing allele frequencies in the patients and control subjects $(\mathrm{OR}=2.333$; 95\% CI: $\left.1.103-4.935 ; p=0.023^{*}\right)$. The frequency of the A allele of TRAF1 was significantly increased in RA compared to control (47.1\% vs. $37.3 \%$ ). Carriers of the A allele were significantly more likely to develop RA $(\mathrm{OR}=1.497$; 95\% CI: 0.976-2.296; $p=0.049$ ) (Table 3).

Table 1. The observed and expected values of the genotype frequencies among the studied groups

\begin{tabular}{|c|c|c|c|c|c|c|c|c|c|}
\hline $\begin{array}{l}\text { TNFAIP3 } \\
\text { rs2203926 }\end{array}$ & Observed & Expected & $\chi^{2}$ & $p$ & $\begin{array}{c}\text { TRAF1 } \\
\text { rs10181488 }\end{array}$ & Observed & Expected & $\chi^{2}$ & $p$ \\
\hline RA cases & & & & & RA cases & & & & \\
\hline $\mathrm{T} / \mathrm{T}$ & 78 & 77.1 & 0.466 & 0.494 & $\mathrm{G} / \mathrm{G}$ & 27 & 29.3 & 0.836 & 0.360 \\
\hline $\mathrm{G} / \mathrm{T}$ & 24 & 25.7 & & & $\mathrm{~A} / \mathrm{G}$ & 57 & 52.3 & & \\
\hline $\mathrm{G} / \mathrm{G}$ & 3 & 2.1 & & & $\mathrm{~A} / \mathrm{A}$ & 21 & 23.3 & & \\
\hline SLE cases & & & & & SLE cases & & & & \\
\hline $\mathrm{T} / \mathrm{T}$ & 66 & 65.0 & 0.649 & 0.420 & $\mathrm{G} / \mathrm{G}$ & 39 & 42.0 & 2.171 & 0.140 \\
\hline $\mathrm{G} / \mathrm{T}$ & 21 & 23.0 & & & $\mathrm{~A} / \mathrm{G}$ & 45 & 39.0 & & \\
\hline $\mathrm{G} / \mathrm{G}$ & 3 & 2.0 & & & $\mathrm{~A} / \mathrm{A}$ & 6 & 9.0 & & \\
\hline Control & & & & & Control & & & & \\
\hline $\mathrm{T} / \mathrm{T}$ & 65 & 65.3 & 0.382 & 0.536 & $\mathrm{G} / \mathrm{G}$ & 27 & 29.5 & 1.466 & 0.225 \\
\hline $\mathrm{G} / \mathrm{T}$ & 10 & 9.3 & & & $\mathrm{~A} / \mathrm{G}$ & 40 & 35.1 & & \\
\hline $\mathrm{G} / \mathrm{G}$ & 0 & 0.3 & & & $\mathrm{~A} / \mathrm{A}$ & 8 & 10.5 & & \\
\hline
\end{tabular}

Qualitative data are expressed using the number and percent and compared using $\chi^{2}$.

* statistically significant at $p \leq 0.05$ 


\section{Association of TNFAIP3 and TRAF1 polymorphisms with autoantibody-positive RA}

The magnitude of association for these loci was increased in those patients who were autoantibody positive; either RF+ or anti-CCP+. TNFAIP3 G allele was signifi-

Table 2. RA patients' clinical characteristics

\begin{tabular}{lc}
\hline Parameter & $\begin{array}{c}\text { RA } \\
(\boldsymbol{n}=\mathbf{1 0 5})\end{array}$ \\
\hline Age (mean \pm SD years) & $41.91 \pm 10.63$ \\
\hline Sex & $27(25.7 \%)$ \\
\hline Male & $78(74.3 \%)$ \\
\hline Female & $56.31 \pm 33.08$ \\
\hline ESR (mm/h) & $24.73 \pm 18.99$ \\
\hline RF positivity & $42(40.0 \%)$ \\
\hline RF (IU/ml) & $15.10(7.0-359.0)$ \\
\hline Anti-CCP positivity & $80(76.2 \%)$ \\
\hline Anti-CCP (IU/ml) & $72.08 \pm 52.95$ \\
\hline DD (mean \pm SD years) & $4.59 \pm 4.58$ \\
\hline Number of tender joints & $7.94 \pm 5.34$ \\
\hline Number of swollen joints & $2.17 \pm 2.46$ \\
\hline DAS28 & $4.59 \pm 0.96$ \\
\hline Disease activity & $38(36.2 \%)$ \\
\hline $\begin{array}{l}\text { Mormally quantitative data are expressed as mean } \pm \text { SD, while abnormally } \\
\text { SD - disease duration }\end{array}$ \\
\hline Severe
\end{tabular}

cantly associated with $\mathrm{RF}+(\mathrm{OR}=2.206, \mathrm{CI}: 1.008-4.825$, $\left.p=0.044^{*}\right)$ and with anti-CCP+ $(16.25 \%$ vs $8.0 \%)$ but without reaching a significant level $(p=0.146)$. TRAF1 A allele was present in $16.25 \%$ of RF+ and only $8.0 \%$ of $\mathrm{RF}-(\mathrm{OR}=2.231, \mathrm{CI}: 0.739-6.735, p=0.146)$ and it was detected in $61.9 \%$ of anti-CCP+ RA patients and none of the anti-CCP negative patients (Tables 4 and 5).

\section{Association of TNFAIP3 and TRAF1 genotypes with RA activity}

In patients with severe RA, the frequencies of TNFAIP3 G/T genotype were significantly increased compared to patients with mild to moderate RA $(36.8 \%$ vs. $14.9 \%)$. Moreover, the frequencies of TRAF1 A/G genotype were significantly increased compared to patients with mild to moderate RA $(60.5 \%$ vs. $50.7 \%)$. The odds ratio of severe rheumatoid arthritis for $\mathrm{A} / \mathrm{G}$ genotype carriers was 5.412 (95\% CI: $0.458-20.091 ; p=0.007$ ) (Table 6).

\section{Clinical characteristics of systemic lupus erythematosus (SLE) patients}

The SLE study group $(n=90)$ consisted of 72 women $(80 \%)$ and 18 men $(20 \%)$. The age (mean \pm SD) of SLE patients was $35.73 \pm 9.84$ years. The mean \pm SD of disease duration was $5.3 \pm 4.04$ years, the mean \pm SD of ESR was $49.47 \pm 36.65 \mathrm{~mm} / \mathrm{h}$, the mean $\pm \mathrm{SD}$ of CRP concentration was $35.48 \pm 36.77 \mathrm{mg} / \mathrm{l}$. Results revealed that $96.7 \%$ of patients were ANA positive, and 69 patients (76.7\%) were anti-dsDNA positive. Lupus nephritis was shown in 45 (50\% of cases) and musculoskeletal manifestations in 48 (53.3\% of cases) (Table 7$)$.

Table 3. Distribution of TNFAIP 3 and TRAF1 alleles and genotypes in rheumatoid arthritis (RA)

\begin{tabular}{lccccc}
\hline & $\begin{array}{c}\text { RA cases } \\
(\boldsymbol{n}=\mathbf{1 0 5})\end{array}$ & $\begin{array}{c}\text { Control } \\
(\boldsymbol{n}=\mathbf{7 5})\end{array}$ & $\chi^{2}$ & $\boldsymbol{p}$ & $\begin{array}{c}\text { Odds ratio } \\
\text { 95\% CI }\end{array}$ \\
\hline SNPs & & & & \\
\hline TNFAIP3 rs2203926 & & & & \\
\hline T/T No. $(\%)$ & $78(74.3 \%)$ & $65(86.7 \%)$ & & & \\
\hline G/T No. $(\%)$ & $24(22.9 \%)$ & $10(13.3 \%)$ & 2.895 & 0.089 & $2.000(0.892-4.486)$ \\
\hline G/G No. $(\%)$ & $3(2.9 \%)$ & $0(0.0 \%)$ & - & - & - \\
\hline G allele No. $(\%)$ & $30(14.3 \%)$ & $10(6.7 \%)$ & $5.143 *$ & $0.023 *$ & $2.333(1.103-4.935)$ \\
\hline TRAF1 rs10181488 & & & & \\
\hline G/G No. $(\%)$ & $27(25.7 \%)$ & $27(36.0 \%)$ & & & $1.425(0.730-2.783)$ \\
\hline A/G No. $(\%)$ & $57(54.3 \%)$ & $40(53.30 \%)$ & 1.079 & 0.299 & $2.625(1.002-6.949)$ \\
\hline A/A No. $(\%)$ & $21(20.0 \%)$ & $8(10.7 \%)$ & $3.887 *$ & $0.047 *$ & $1.497(0.976-2.296)$ \\
\hline A allele No. $(\%)$ & $99(47.1 \%)$ & $56(37.3 \%)$ & 3.434 & $0.04)^{*}$ & \\
\hline
\end{tabular}


Table 4. Genotype frequencies in rheumatoid factor positive rheumatoid arthritis, relative to negative

\begin{tabular}{|c|c|c|c|c|c|}
\hline & $\begin{array}{c}\mathrm{RF}^{+} \text {cases } \\
(n=42)\end{array}$ & $\begin{array}{c}\mathbf{R F}^{-} \\
(n=63)\end{array}$ & $\chi^{2}$ & $p$ & $\begin{array}{c}\text { Odds ratio } \\
95 \% \text { CI }\end{array}$ \\
\hline \multicolumn{6}{|l|}{ SNPs } \\
\hline \multicolumn{6}{|c|}{ TNFAIP3 rs2203926 } \\
\hline T/T No. $(\%)$ & $28(66.7 \%)$ & $50(79.4 \%)$ & & & \\
\hline G/T No. (\%) & $11(26.2 \%)$ & $13(20.6 \%)$ & 0.767 & 0.381 & $1.511(0.598-3.817)$ \\
\hline G/G No. $(\%)$ & $3(7.1 \%)$ & $0(0.0 \%)$ & - & - & - \\
\hline G allele No. $(\%)$ & $17(20.2 \%)$ & $13(10.3 \%)$ & $4.051 *$ & $0.044 *$ & $2.206(1.008-4.825)$ \\
\hline \multicolumn{6}{|c|}{ TRAF1 rs10181488 } \\
\hline G/G No. (\%) & $0(0.0 \%)$ & $27(42.9 \%)$ & & & \\
\hline A/G No. (\%) & $30(71.4 \%)$ & $27(42.9 \%)$ & - & - & - \\
\hline A/A No. (\%) & $12(28.6 \%)$ & $9(14.3 \%)$ & - & - & - \\
\hline A allele No. (\%) & $54(64.3 \%)$ & $45(35.7 \%)$ & $16.511 *$ & $<0.001^{*}$ & $3.240(1.821-5.765)$ \\
\hline
\end{tabular}

$R F^{+}-$rheumatoid factor positive; $R F^{-}$-rheumatoid factor negative

Table 5. Genotype frequencies in anti-CCP positive rheumatoid arthritis, relative to anti-CCP negative

\begin{tabular}{lccccc}
\hline & $\begin{array}{c}\text { Anti-CCP+ } \\
(\boldsymbol{n}=\mathbf{8 0})\end{array}$ & $\begin{array}{c}\text { Anti-CCP- } \\
(\boldsymbol{n}=\mathbf{2 5})\end{array}$ & $\chi^{2}$ & $\boldsymbol{p}$ & $\begin{array}{c}\text { Odds ratio } \\
\text { 95\% CI }\end{array}$ \\
\hline SNPs & & & & \\
\hline TNFAIP3 rs2203926 & & & & \\
\hline T/T No. $(\%)$ & $57(71.3 \%)$ & $21(84.0 \%)$ & & & $-1.842(0.564-6.021)$ \\
\hline G/T No. $(\%)$ & $20(25.0 \%)$ & $4(16.0 \%)$ & 1.043 & 0.307 & - \\
\hline G/G No. $(\%)$ & $3(3.8 \%)$ & $0(0.0 \%)$ & - & - & - \\
\hline G allele No. $(\%)$ & $26(16.25 \%)$ & $4(8.0 \%)$ & 2.117 & 0.146 & - \\
\hline TRAF1 rs10181488 & & & & - & - \\
\hline G/G No. $(\%)$ & $2(2.5 \%)$ & $25(100.0 \%)$ & - & - & - \\
\hline A/G No. $(\%)$ & $57(71.3 \%)$ & $0(0.0 \%)$ & - & - & - \\
\hline A/A No. $(\%)$ & $21(26.3 \%)$ & $0(0.0 \%)$ & - & - & - \\
\hline A allele No. $(\%)$ & $99(61.9 \%)$ & $0(0.0 \%)$ & - & - & - \\
\hline
\end{tabular}

Qualitative data are expressed using the number and percent and compared using $\chi^{2}$.

* statistically significant at $p \leq 0.05$

Table 6. Association of TNFAIP3 and TRAF1 genotypes with RA activity

\begin{tabular}{|c|c|c|c|c|c|}
\hline & $\begin{array}{l}\text { Mild to moderate } \\
\quad(n=67)\end{array}$ & $\begin{array}{c}\text { Severe } \\
(n=38)\end{array}$ & $\chi^{2}$ & $p$ & $\begin{array}{c}\text { Odds ratio } \\
95 \% \mathrm{CI}\end{array}$ \\
\hline \multicolumn{6}{|l|}{ SNPs } \\
\hline \multicolumn{6}{|c|}{ TNFAIP3 rs2203926 } \\
\hline T/T No. (\%) & $57(85.1 \%)$ & $21(55.3 \%)$ & & & \\
\hline G/T No. $(\%)$ & $10(14.9 \%)$ & $14(36.8 \%)$ & $8.034^{*}$ & $0.005^{*}$ & $3.800(1.465-9.859)$ \\
\hline G/G No. (\%) & $0(0.0 \%)$ & $3(7.9 \%)$ & - & - & - \\
\hline G allele No. (\%) & $10(7.5 \%)$ & $20(26.3 \%)$ & $14.077^{*}$ & $<0.001^{*}$ & $4.429(1.946-10.076)$ \\
\hline \multicolumn{6}{|c|}{ TRAF1 rs10181488 } \\
\hline G/G No. $(\%)$ & $24(35.8 \%)$ & $3(7.9 \%)$ & & & \\
\hline A/G No. $(\%)$ & $34(50.7 \%)$ & $23(60.5 \%)$ & $7.329^{*}$ & $0.007 *$ & $5.412(1.458-20.091)$ \\
\hline A/A No. (\%) & $9(13.4 \%)$ & $12(31.6 \%)$ & $11.650^{*}$ & $0.001 *$ & $10.667(2.431-46.812)$ \\
\hline A allele No. (\%) & $52(38.8 \%)$ & $47(61.8 \%)$ & $11.508^{*}$ & $0.001 *$ & $2.680(1.505-4.772)$ \\
\hline
\end{tabular}




\section{Association of TNFAIP3 (rs2230926) and TRAF1 (rs10818488) polymorphism genotypes with SLE susceptibility}

Genotyping SNPs was done by using the Step One real-time PCR system (Applied Biosystems-Life Technologies, Carlsbad, California, USA). Full genotype distribution and allele frequency data are shown in Table 5. In SLE patients, the frequencies of the risk allele TNFAIP3 G were significantly increased compared to the control group $\left(\mathrm{OR}=2.471 ; 95 \% \mathrm{CI}: 1.154-5.288 ; p=0.017^{*}\right)$. The frequencies was $(15 \%)$ compared with an allele frequency of $(6.7 \%)$ in control individuals. The polymorphism of

Table 7. SLE patients' clinical characteristics

\begin{tabular}{lc}
\hline Parameter & $\begin{array}{c}\text { SLE } \\
(\boldsymbol{n}=\mathbf{9 0})\end{array}$ \\
\hline Age (mean \pm SD years) & $35.73 \pm 9.84$ \\
\hline Sex & $18(20.0 \%)$ \\
\hline Male & $72(80.0 \%)$ \\
\hline Female & $49.47 \pm 36.65$ \\
\hline CRP (mg/ml) & $35.48 \pm 36.77$ \\
\hline DD (years) & $5.31 \pm 4.04$ \\
\hline ANA positivity & $87(96.7 \%)$ \\
\hline Anti DNA positivity & $69(76.7 \%)$ \\
\hline Lupus nephritis & $45(50.0 \%)$ \\
\hline Musculoskeletal & $48(53.3 \%)$ \\
\hline Neuropsychiatric & $6(6.7 \%)$ \\
\hline Vascular & $15(16.7 \%)$ \\
\hline SLEDAI & $19.67 \pm 14.54$ \\
\hline
\end{tabular}

the TRAF1 rs10818488SNP analyzed did not exhibit any statistical significant difference in the frequencies of genotypes or alleles in patients and controls, A allele (OR = 0.778; 95\% CI: 0.493-1.227; $p=0.280$ ) (Table 8).

\section{Association of TNFAIP3 and TRAF1 polymorphisms with autoantibody-positivity}

As ANA positivity was observed 87/90 SLE patients, only 3 patients were negative for this autoantibody so the magnitude of association of TNFAIP 3 or TRAF1 could not be evaluated. Anti-dsDNA was present in 69 SLE cases and showed no association with the $\mathrm{G}$ allele nor the $\mathrm{G} / \mathrm{T}$ genotype ( $p=0.586$ and 0.882 , respectively). The results were obtained for A allele of TRAF1 (OR $=0.788$, $p=0.52$ ) (Table 9).

\section{Association of TNFAIP3 and TRAF1 polymorphisms with activity in SLE}

In patients with severe SLE, the frequencies of TNFAIP3 G/T genotype were not associated with severity (16/7 in severe cases vs. $33.3 \%$ in mild to moderate flare). The odds ratio of severe SLE for G/T genotype carriers was 0.429 (95\% CI: $0.158-1.164 ; p=0.092$ ). Moreover, the frequencies of TRAF1 A/ allele were also of similar distribution between severe SLE compared to patients with mild to moderate SLE (32.4\% vs. 30.8\%) (Table 10).

\section{Relation between TNFAIP3 and TRAF1 with different parameters in RA and SLE patients}

To elucidate the relationship between studied SNPs and RA susceptibility, we conducted further statistical analyses. We analyzed the interactions between the studied genotypes and known disease criteria. ESR, DAS28 and swollen joint count showed a significant interaction with

Table 8. Distribution of TNFAIP3 and TRAF1 alleles and genotypes in SLE

\begin{tabular}{|c|c|c|c|c|c|}
\hline & $\begin{array}{l}\text { SLE cases } \\
\quad(n=90)\end{array}$ & $\begin{array}{l}\text { Control } \\
(n=75)\end{array}$ & $\chi^{2}$ & $p$ & $\begin{array}{c}\text { Odds ratio } \\
95 \% \text { CI }\end{array}$ \\
\hline \multicolumn{6}{|l|}{ SNPs } \\
\hline \multicolumn{6}{|c|}{ TNFAIP3 rs2203926 } \\
\hline T/T No. (\%) & $66(73.3 \%)$ & $65(86.7 \%)$ & & & \\
\hline G/T No. (\%) & $21(23.3 \%)$ & $10(13.3 \%)$ & 3.039 & 0.081 & $2.068(0.904-4.730)$ \\
\hline G/G No. (\%) & $3(3.3 \%)$ & $0(0.0 \%)$ & - & - & - \\
\hline G allele No. (\%) & $27(15.0 \%)$ & $10(6.7 \%)$ & $5.707 *$ & $0.017^{*}$ & $2.471(1.154-5.288)$ \\
\hline \multicolumn{6}{|c|}{ TRAF1 rs10181488 } \\
\hline G/G No. (\%) & $39(43.3 \%)$ & $27(36.0 \%)$ & & & \\
\hline A/G No. (\%) & $45(50.0 \%)$ & $40(53.3 \%)$ & 0.569 & 0.451 & $0.779(0.407-1.492)$ \\
\hline A/A No. (\%) & $6(6.7 \%)$ & $8(10.7 \%)$ & 1.237 & 0.266 & $0.519(0.162-1.667)$ \\
\hline A allele No. (\%) & $57(31.7 \%)$ & $56(37.3 \%)$ & 1.167 & 0.280 & $0.778(0.493-1.227)$ \\
\hline
\end{tabular}


Table 9. Genotype frequencies in anti-dsDNA positive SLE

\begin{tabular}{|c|c|c|c|c|c|}
\hline & Anti-dsDNA+ $(n=69)$ & $\begin{array}{l}\text { Anti d -ve } \\
\quad(n=21)\end{array}$ & $\chi^{2}$ & $p$ & $\begin{array}{c}\text { Odds ratio } \\
95 \% \text { CI }\end{array}$ \\
\hline \multicolumn{6}{|l|}{ SNPs } \\
\hline \multicolumn{6}{|c|}{ TNFAIP3 rs2203926 } \\
\hline T/T No. (\%) & $51(73.9 \%)$ & $15(71.4 \%)$ & & & \\
\hline G/T No. $(\%)$ & $15(21.7 \%)$ & $6(28.6 \%)$ & 0.297 & 0.586 & $0.735(0.243-2.227)$ \\
\hline G/G No. (\%) & $3(4.3 \%)$ & $0(0.0 \%)$ & - & - & - \\
\hline G allele No. (\%) & $21(15.2 \%)$ & $6(14.3 \%)$ & 0.022 & 0.882 & $1.077(0.404-2.873)$ \\
\hline \multicolumn{6}{|c|}{ TRAF1 rs10181488 } \\
\hline G/G No. (\%) & $33(47.8 \%)$ & $6(28.6 \%)$ & & & \\
\hline A/G No. (\%) & $30(43.5 \%)$ & $15(71.4 \%)$ & 3.590 & 0.058 & $0.364(0.125-1.058)$ \\
\hline A/A No. (\%) & $6(8.7 \%)$ & $0(0.0 \%)$ & - & - & - \\
\hline A allele No. (\%) & $42(30.4 \%)$ & $15(35.7 \%)$ & 0.415 & 0.520 & $0.788(0.380-1.631)$ \\
\hline
\end{tabular}

Table 10. Genotype frequencies with SLE severity

\begin{tabular}{|c|c|c|c|c|c|}
\hline & $\begin{array}{l}\text { Mild to moderate flare } \\
\qquad(n=36)\end{array}$ & $\begin{array}{l}\text { Severe flare } \\
\quad(n=54)\end{array}$ & $\chi^{2}$ & $p$ & $\begin{array}{c}\text { Odds ratio } \\
\text { 95\% CI }\end{array}$ \\
\hline \multicolumn{6}{|l|}{ SNPs } \\
\hline \multicolumn{6}{|c|}{ TNFAIP3 rs2203926 } \\
\hline T/T No. (\%) & $24(66.7 \%)$ & $42(77.8 \%)$ & & & \\
\hline G/T No. (\%) & $12(33.3 \%)$ & $9(16.7 \%)$ & 2.836 & 0.092 & $0.429(0.158-1.164)$ \\
\hline G/G No. (\%) & $0(0.0 \%)$ & $3(5.6 \%)$ & - & - & - \\
\hline G allele No. $(\%)$ & $12(15.4 \%)$ & $15(14.7 \%)$ & 0.016 & 0.899 & $0.948(0.416-2.161)$ \\
\hline \multicolumn{6}{|c|}{ TRAF1 rs10181488 } \\
\hline G/G No. (\%) & $12(33.3 \%)$ & $27(50.0 \%)$ & & & \\
\hline A/G No. (\%) & $24(66.7 \%)$ & $21(38.9 \%)$ & $4.344 *$ & $0.037 *$ & $0.389(0.159-0.954)$ \\
\hline A/A No. $(\%)$ & $0(0.0 \%)$ & $6(11.1 \%)$ & - & - & - \\
\hline A allele No. (\%) & $24(30.8 \%)$ & $33(32.4 \%)$ & 0.051 & 0.821 & $1.076(0.570-2.031)$ \\
\hline
\end{tabular}

TNFAIP3. ESR, autoantibodies, DAS28 and tender joint counts with TRAF1 (Tables 11 and 12). This was done also with SLE, as shown in Tables 13 and 14.

\section{Discussion}

In this study, we found that TNFAIP3 rs2230926 G/T genotype displayed a 2 fold increased risk of RA and this result confirmed an association of this polymorphism with the susceptibility to RA in Egypt. The G allele was consistently less common in controls than cases. Several studies in different populations had confirmed this association; Shimane et al. on Japanese populations [23], Zhang et al. on Chinese patients [24] and Musone et al. [25] who examined European patients with multiple autoimmune diseases, including RA.
RA is a heterogeneous disease with a significant discrepancy in the phenotype as demonstrated by differences in autoantibody positivity. Anti-CCP has gained much attention as current data suggest that anti-CCP positive and negative RA may have different genetic risk factors [26]. Though the specificity of RF has little diagnostic significance for RA, it is an effective indicator of RA activity and a high titer of RF suggests a poor prognosis of RA [27]. We tested whether rs2230926 in TNFAIP3 was associated with autoantibody-positive RA and we observed an increase in the frequency of the G allele in autoantibody positive cases as regards RF (with a significance) and antiCCP (without significance) compared to those who are autoantibodies negative. Therefore, harboring the $\mathrm{G}$ allele may be a risk factor. Several risk alleles for rheumatoid arthritis have been identified in gene regions containing 
Table 11. Relation between TNFAIP3 with different parameters in the RA group

\begin{tabular}{|c|c|c|c|c|}
\hline \multirow[t]{2}{*}{ Parameter } & \multicolumn{3}{|c|}{ TNFAIP3 } & \multirow[t]{2}{*}{$p$} \\
\hline & $\begin{array}{c}\mathbf{T} / \mathbf{T} \\
(n=78)\end{array}$ & $\begin{array}{c}\text { G/T } \\
(n=24)\end{array}$ & $\begin{array}{c}\text { G/G } \\
(n=3)\end{array}$ & \\
\hline Age & $41.0^{c} \pm 10.15$ & $42.63^{c} \pm 10.64$ & $60.0^{\mathrm{ab}} \pm 8.66$ & $0.008^{*}$ \\
\hline $\mathrm{DD}$ & $4.74 \pm 5.09$ & $3.75 \pm 2.14$ & $7.67 \pm 4.04$ & 0.329 \\
\hline $\operatorname{ESR}\left(1^{\text {st }} \mathrm{hr}\right.$.) & $46.88^{\mathrm{b}} \pm 23.93$ & $85.75^{\mathrm{a}} \pm 42.51$ & $66.0 \pm 6.93$ & $<0.001^{*}$ \\
\hline $\mathrm{CRP}(\mathrm{mg} / \mathrm{l})$ & $24.62 \pm 19.50$ & $26.40 \pm 17.99$ & $14.12 \pm 13.75$ & 0.574 \\
\hline $\mathrm{RF}(\mathrm{IU} / \mathrm{ml})$ & $15.05(7.0-359.0)$ & $18.75(8.0-189.0)$ & $27.0(27.0-41.20)$ & 0.285 \\
\hline Anti-CCP (U/ml) & $64.59^{\mathrm{b}} \pm 48.14$ & $95.34^{\mathrm{a}} \pm 59.73$ & $80.87 \pm 81.87$ & $0.042^{*}$ \\
\hline Disease activity score (DAS28) & $4.38^{\mathrm{b}} \pm 0.94$ & $5.20^{\mathrm{a}} \pm 0.78$ & $5.24 \pm 0.45$ & $<0.001^{*}$ \\
\hline Tender joints count & $7.44^{\mathrm{b}} \pm 5.36$ & $10.0^{\mathrm{a}} \pm 5.11$ & $4.67 \pm 1.15$ & 0.066 \\
\hline Swollen joints count & $1.77^{\mathrm{c}} \pm 1.35$ & $2.75^{\mathrm{c}} \pm 3.67$ & $8.0^{\mathrm{ab}} \pm 5.20$ & $<0.001^{*}$ \\
\hline
\end{tabular}

Table 12. Relation between TRAF1 with different parameters in the RA group

\begin{tabular}{|c|c|c|c|c|}
\hline \multirow[t]{2}{*}{ Parameter } & \multicolumn{3}{|c|}{ TRAF1 } & \multirow[t]{2}{*}{$p$} \\
\hline & $\begin{array}{c}\text { G/G } \\
(n=27)\end{array}$ & $\begin{array}{c}\mathbf{A} / \mathbf{G} \\
(n=57)\end{array}$ & $\begin{array}{c}\text { A/A } \\
(n=21)\end{array}$ & \\
\hline Age & $40.52 \pm 7.54$ & $43.54 \pm 12.37$ & $39.29 \pm 8.22$ & 0.215 \\
\hline DD & $5.56 \pm 6.28$ & $4.48 \pm 4.32$ & $3.67 \pm 1.74$ & 0.355 \\
\hline $\operatorname{ESR}\left(1^{\text {st }} \mathrm{hr}.\right)$ & $38.56^{b} \pm 20.42$ & $66.77^{\mathrm{ac}} \pm 36.49$ & $50.76^{\mathrm{b}} \pm 25.49$ & $0.001 *$ \\
\hline $\mathrm{CRP}(\mathrm{mg} / \mathrm{l})$ & $22.81 \pm 17.66$ & $23.31 \pm 18.24$ & $31.04 \pm 22.05$ & 0.235 \\
\hline $\mathrm{RF}(\mathrm{IU} / \mathrm{ml})$ & $12.0^{\mathrm{bc}}(7.0-19.90)$ & $24.0^{\mathrm{a}}(7.19-359.0)$ & $24.31^{\mathrm{a}}(7.31-102.0)$ & $<0.001^{*}$ \\
\hline Anti-CCP (U/ml) & $33.16^{c} \pm 34.14$ & $94.50^{\mathrm{ac}} \pm 53.86$ & $61.28^{\mathrm{ab}} \pm 36.62$ & $<0.001^{*}$ \\
\hline Disease activity score (DAS28) & $4.20^{\mathrm{bc}} \pm 0.76$ & $4.70^{\mathrm{a}} \pm 0.96$ & $4.79^{\mathrm{a}} \pm 1.09$ & $0.048^{*}$ \\
\hline Tender joints count & $6.48^{c} \pm 4.29$ & $7.79 \pm 4.47$ & $10.24^{\mathrm{a}} \pm 7.76$ & $0.050^{*}$ \\
\hline Swollen joints count & $1.96 \pm 2.19$ & $2.14 \pm 2.25$ & $2.52 \pm 3.28$ & 0.731 \\
\hline
\end{tabular}

Table 13. Relation between TNFAIP3 with different parameters in the SLE group

\begin{tabular}{|c|c|c|c|c|}
\hline \multirow[t]{2}{*}{ Parameter } & \multicolumn{3}{|c|}{ TNFAIP3 } & \multirow[t]{2}{*}{$p$} \\
\hline & $\begin{array}{c}T / T \\
(n=66)\end{array}$ & $\begin{array}{c}\text { G/T } \\
(n=21)\end{array}$ & $\begin{array}{c}\mathbf{G} / \mathbf{G} \\
(n=3)\end{array}$ & \\
\hline Age & $28.91^{\mathrm{bc}} \pm 8.77$ & $38.43^{\mathrm{a}} \pm 8.52$ & $47.0^{\mathrm{a}} \pm 0.0$ & $<0.001 *$ \\
\hline DD & $5.45 \pm 4.31$ & $4.51 \pm 3.21$ & $8.0 \pm 0.0$ & 0.332 \\
\hline $\operatorname{ESR}\left(1^{\text {st }} \mathrm{hr}.\right)$ & $49.60 \pm 38.15$ & $49.0 \pm 35.29$ & $50.0 \pm 0.0$ & 0.998 \\
\hline CRP (mg/l) & $38.41 \pm 38.04$ & $30.97 \pm 33.15$ & $2.50 \pm 0.0$ & 0.209 \\
\hline
\end{tabular}

TNFAIP3 and TRAF1 genes. On the contrary to our findings, results in Caucasian RA patients have demonstrated that the TNFAIP3 variant led to an increased risk of RA in negative anti-CCP antibody patients compared with a positive anti-CCP antibody [26]. These new findings and data from complementary candidate gene studies [28] indicate how series of variations together make up the genetic risk for rheumatoid arthritis, and they show how diverse patterns of genetic risk factors have emerged for subsets of disease positive and negative for anti-CCP or RF. 
Table 14. Relation between TRAF1 with different parameters in the SLE group

\begin{tabular}{|c|c|c|c|c|}
\hline \multirow[t]{2}{*}{ Parameter } & \multicolumn{3}{|c|}{ TRAF1 } & \multirow[t]{2}{*}{$p$} \\
\hline & $\begin{array}{c}\text { G/G } \\
(n=39)\end{array}$ & $\begin{array}{c}\mathbf{A} / \mathbf{G} \\
(n=45)\end{array}$ & $\begin{array}{c}\mathbf{A} / \mathbf{A} \\
(n=6)\end{array}$ & \\
\hline Age & $30.08 \pm 9.44$ & $32.80 \pm 10.49$ & $34.50 \pm 6.02$ & 0.352 \\
\hline DD & $5.0 \pm 2.48$ & $5.69 \pm 5.20$ & $4.50 \pm 1.64$ & 0.649 \\
\hline ESR ( $\left(1^{\text {st }} \mathrm{hr}\right.$.) & $36.43^{\mathrm{b}} \pm 29.15$ & $58.57^{\mathrm{a}} \pm 40.22$ & $66.0 \pm 28.48$ & $0.010^{*}$ \\
\hline CRP (mg/l) & $33.45 \pm 40.57$ & $41.36^{c} \pm 33.80$ & $4.49^{b} \pm 1.66$ & 0.061 \\
\hline
\end{tabular}

In our study we stratified the studied patients according to ESR and CRP levels among other clinical and laboratory parameters. Since RA is a systemic vascular inflammatory disease, ESR is considered a relatively reliable indicator of the acute phase reaction of inflammation although its specificity and sensitivity are not perfect for the diagnosis of RA. The CRP level is more correlated with the degree of inflammation in RA. C-reactive protein is not only an inflammatory marker, but may also induce a proinflammatory effect by activating monocyte chemoattractant protein and RA disease activity was positively correlated with the CRP level [29]. Our study found a significant difference in ESR $(p<0.001)$ and not with CRP $(p=0.574)$ across the TNFAIP3 SNPs examined.

The TRAF1 gene encodes the tumor necrosis factor (TNF) receptor associated factor 1, which is considered to be associated with multiple TNF receptor family members and regulates cytokine signaling networks including TNF- $\alpha$ by binding several protein kinases and adaptor proteins. Our results clarified that TRAF1 A/G genotype displayed a 2.625 fold increased risk of RA and this result suggests an association of the polymorphism with RA. These results agree with Zervou et al. [30] who suggested that this region is a RA associated region. On the contrary a genome-wide study performed by Burton et al. [31] failed to identify this region as a candidate for RA. Another study carried out by Ahmediou et al. [32] in the Iranian population suggested lack of association between TRAF1 and RA. Therefore, rs10818488 TRAF1 might not be a genius allele related to RA in Asians and this can be explained by distinct population specific differences in the prevalence of this allele. The Iranian population is composed of different ethnic groups, so identifying alleles that have a lower frequency in this population is hardly possible.

Also we showed that (rs10818488) TRAF1 was not only strongly associated with RA but also with autoantibody positive RA. This agrees with the results obtained by Kurreeman et al. [33]. Most RA patients harbor this antibody in their serum and synovial fluid during early stages of RA disease, even before the first symptoms appear [34]. Kroot et al. [35] reported that anti-CCP antibody was correlated with RA activity. Studies by Kamradt et al. [36] and Agrawal et al. [37] suggest that anti-CCP antibody can be used as a marker of RA activity. Glasnovic et al. [38] found that anti-CCP antibody could predict RA condition and that its titer was related to radiological joint lesions. The anti-CCP antibody-positive rate among the $\mathrm{AA}+\mathrm{AG}$ patients was higher than among the GG patients $(p<0.001)$, suggesting that the AA+GA genotype may be a risk factor for RA. All anti-CCP negative cases (100\%) were harboring the GG phenotype and this indicates that this phenotype may be a protective one. The above result was indirectly supported by Viatte et al. in anti-CCP antibody negative patients [39].

In the present study, it was found that in the Egyptian population, TNFAIP3 T/G genotype displayed an increased risk of SLE and this result confirmed an association of the polymorphism with SLE. The G allele was consistently less common in controls than in cases. Several studies had confirmed the association in European populations; Graham et al. [40], Musone et al. [41], and this was found also in both Chinese populations; Han et al. Cai et al. [42, 43] and Zhong et al. [44] and Japanese populations; Kawasaki et al. [45]. Interestingly, the minor alleles of rs13192841 and rs6922466 were protective for SLE, while the minor allele of rs2230926 conferred an increased SLE risk [41].

Lodolce et al. [46] performed the first study examining the association between TNFAIP3/A20 SNPs and SLE in the African-American population, identifying an African ancestry-specific risk allele at rs2230926 that was associated with the increased SLE risk. In a study by Adrianto et al. [8] who examined 5 populations of different ancestry (African-American, Asian (Korean), European, African-American Gullah and Hispanic), they found a strong association between a heightened risk of SLE and rs2230926 in Europeans and Asians.

We next aimed at testing whether rs2230926 in TNFAIP3 was associated with autoantibody positivity either 
ANA+ or anti-dsDNA+. ANA was found to be positive in $96.7 \%$ of cases, so we could not establish a comparative association. However, the frequency of rs $2230926 \mathrm{G}$ allele was not significantly increased in SLE with anti-dsDNA+ positive; as compared with negative cases. This finding is not in agreement with Cai et al. [43] who showed that the rs2230926 was significantly associated with arthritis, autoantibodies of ANA, Anti-Smith (Anti-Sm) and Anti-dsDNA, which highlighted the importance of the regulation of innate and adaptive immunity in the etiology of SLE.

We also analyzed whether the SNP in TNFAIP3 was involved in SLE activity. SLE patients with G allele have no association between severe flare as compared to the mild to moderate patients carried the same allele. We also did not find any significant difference in the studied SNPs of TNFAIP3 in relation to patient's criteria (disease duration, ESR and CRP). Thus we can suggest that these potentially SLE susceptibility genes are related to SLE occurrence and not progression, which is expected through their role in enhancement of autoreactivity. But still, results should be interpreted carefully because of low study power to reveal small effects and few cases to investigate disease heterogeneity by different environmental and pathological variables. Additional restriction of our small sample size was the inability to assess SNP-SNP interactions or linkage disequilibrium between polymorphisms within the same pathway and different pathways, which we recommend in upcoming studies.

In the present study, we observed that no association was found between TRAF1 polymorphism (rs10818488) and susceptibility to SLE. This goes in accordance with Nishimoto et al. [12] on Japanese populations, Palomino-Morales et al. [47] on Colombian patients with SLE and Kurreeman et al. [11] in the Dutch population. Contrary to our results, two studies in Caucasian populations carried out by Kurreeman et al. [11] reported an association of SNP rs10818488 of TRAF1 with the risk of SLE in the Greek population and Spanish populations and Zervou et al. [48] in Turkish populations.

In our study, we showed that TRAF1 A allele did not exhibit any significant association with anti-dsDNA+ and SLE activity.

In conclusion, our findings demonstrated an association between TNFAIP3 (rs2230926) and Egyptian patients with SLE and RA. It indicates a global risk of this polymorphism. Also our study independently replicated and confirmed the strong association of SNP (rs10818488) of TRAF1 with the risk of RA in the Egyptian population, while the association between this locus and SLE could not be observed. Nevertheless, our results could offer a starting point for additional analysis of the role of TNFAIP3 and TRAF1 gene polymorphisms on the risk of RA and SLE in the Egyptian population to explain the molecular pathways through in vitro and in vivo future studies for the remaining SNPs.
The authors declare no conflict of interest.

\section{References}

1. Majithia V, Geraci SA (2007): Rheumatoid arthritis: diagnosis and management. Am J Med 120: 936-939.

2. Gabriel SE, Michaud K (2009): Epidemiological studies in incidence, prevalence, mortality, and comorbidity of the rheumatic diseases. Arthritis Res Ther 11: 229.

3. Krishnan S, Chowdhury B, Juang Y-T, Tsokos GC (2007): Overview of the pathogenesis of systemic lupus erythematosus. In: Tsokos GC, Gordon C, Smolen JS, editors. Systemic lupus erythematosus: acompanion to Rheumatology. Mosby Inc; Philadelphia; 55-63.

4. Davidson A, Diamond B (2001): Autoimmune diseases. N Engl J Med 345: 340-350.

5. Suzuki A, Kochi Y, Okada Y, Yamamoto K (2011): Insight from genome-wide association studies in rheumatoid arthritis and multiple sclerosis. FEBS Lett 585: 3627-3632.

6. Harley IT, Kaufman KM, Langefeld CD, et al. (2009): Genetic susceptibility to SLE: new insights from fine mapping and genome-wide association studies. Nat Rev Genet 10: 285-290.

7. Coornaert B, Carpentier I, Beyaert R (2009): A20: central gatekeeper in inflammation and immunity. J Biol Chem 284: 8217-8221.

8. Adrianto I, Wen F, Templeton A, et al. (2011): Association of a functional variant downstream of TNFAIP3 with systemic lupus erythematosus. Nat Genet 43: 253-258.

9. Wajant H, Henkler F, Scheurich P (2001): The TNF-receptor-associated factor family: scaffold molecules for cytokine receptors, kinases and their regulators. Cell Signal 13: 389400 .

10. Sabbagh L, Srokowski CC, Pulle G, et al. (2006): A critical role for TNF receptor-associated factor 1 and Bim down-regulation in CD8 memory T cell survival. Proc Natl Acad Sci U S A 103: 18703-18708.

11. Kurreeman FA, Goulielmos GN, Alizadeh BZ, et al. (2010): The TRAF1-C5 region on chromosome 9q33 is associated with multiple autoimmune diseases. Ann Rheum Dis 69: 696699.

12. Nishimoto K, Kochi Y, Ikari K, et al. (2010): Association study of TRAF1-C5 polymorphisms with susceptibility to rheumatoid arthritis and systemic lupus erythematosus in Japanese. Ann Rheum Dis 69: 368-373.

13. Lee YH, Harley JB, Nath SK (2006): Meta-analysis of TNF-alpha promoter $-308 \mathrm{~A} / \mathrm{G}$ polymorphism and SLE susceptibility. Eur J Hum Genet 14: 364-371.

14. Neogi T, Aletaha D, Silman AJ, et al. (2010): The 2010 American College of Rheumatology/European League Against Rheumatism classification criteria for rheumatoid arthritis. Arthritis Rheum 62: 2582-2591.

15. Van der Heijde DM, van t Hof MA, van Riel PL, et al. (1990): Judging disease activity in clinical practice in rheumatoid arthritis: first step in the development of a disease activity score. Ann Rheum Dis 49: 916-920.

16. Petri M, Orbai AM, Alarcon GS, et al. (2012): Derivation and validation of the Systemic Lupus International Collaborating Clinics classification criteria for systemic lupus erythematosus. Arthritis Rheum 64: 2677-2686.

17. Griffiths B, Mosca M, Gordon C (2005): Assessment of patients with systemic lupus erythematosus and the use of lupus 
disease activity indices. Best Pract Res Clin Rheumatol 19: 685-708.

18. Masi AT, Aldag JC, Sipes J (2001): Do elevated levels of serum C-reactive protein predict rheumatoid arthritis in men: correlations with pre-RA status and baseline positive rheumatoid factors. J Rheumatol 28: 2359-2361.

19. Thammanichanond D, Kunakorn M, Kitiwanwanich S, et al. (2005): Raising rheumatoid factor cutoff helps distinguish rheumatoid arthritis. Asian Pac J Allergy Immunol 23: 165168.

20. Suzuki K, Sawada T, Murakami A, et al. (2003): High diagnostic performance of ELISA detection of antibodies to citrullinated antigens in rheumatoid arthritis. Scand J Rheumatol 32: 197-204.

21. QIAamp DNA Blood Mini Kit. Available from: www.qiagen. com.

22. Malkki M, Petersdorf EW (2012): Genotyping of single nucleotide polymorphisms by 5 ' nuclease allelic discrimination. Methods Mol Biol 882: 173-182.

23. Shimane K, Kochi Y, Horita T, et al. (2010): The association of a nonsynonymous single-nucleotide polymorphism in TNFAIP3 with systemic lupus erythematosus and rheumatoid arthritis in the Japanese population. Arthritis Rheum 62: 574579.

24. Zhang X, Li W, Jiang L, et al. (2013): The association of single nucleotide polymorphisms in TNFAIP3 with rheumatoid arthritis in the Chinese population. Centr Eur J Immunol 38: 214-220.

25. Musone SL, Taylor KE, Nititham J, et al. (2011): Sequencing of TNFAIP3 and association of variants with multiple autoimmune diseases. Genes Immun 12: 176-182.

26. Van Gaalen F, Ioan-Facsinay A, Huizinga TW, Toes RE (2005): The devil in the details: the emerging role of anticitrulline autoimmunity in rheumatoid arthritis. J Immunol 175: 5575-5580.

27. Tedesco A, D'Agostino D, Soriente I, et al. (2009): A new strategy for the early diagnosis of rheumatoid arthritis: a combined approach. Autoimmun Rev 8: 233-237.

28. Perdigones N, Lamas JR, Vigo AG, et al. (2009): 6q23 polymorphisms in rheumatoid arthritis Spanish patients. Rheumatology (Oxford) 48: 618-621.

29. Yeh ET (2004): CRP as a mediator of disease. Circulation 109 (21 Suppl 1): II: 11-14.

30. Zervou MI, Sidiropoulos P, Petraki E, et al. (2008): Association of a TRAF1 and a STAT4 gene polymorphism with increased risk for rheumatoid arthritis in a genetically homogeneous population. Hum Immunol 69: 567-571.

31. Burton PR, Clayton DG, Cardon LR, et al. (2007): The Wellcome Trust Case Control Consortium, Genome-wide association study of 14,000 cases of seven common diseases and 3000 shared controls. Nature 447: 661-678.

32. Ahmadlou S, Akhiani M, Salimzadeh A, et al. (2014): Lack of Association between Single Nucleotide Polymorphism rs10818488 in TRAF1/C5 Region and Rheumatoid Arthritis in Iranian Population. Iran J Allergy Asthma Immunol 13: 19-25.

33. Kurreeman FA, Padyukov L, Marques RB, et al. (2007): A candidate gene approach identifies the TRAF1/C5 region as a risk factor for rheumatoid arthritis. PLoS Med 4: e278.

34. Jansen AL, van der Horst-Bruinsma I, van Schaardenburg D, et al. (2011): Rheumatoid factor and antibodies to cyclic citrullinated Peptide differentiate rheumatoid arthritis from undifferentiated polyarthritis in patients with early arthritis. J Rheumatol 29: 2074-2076.

35. Kroot EJ, de Jong BA, van Leeuwen MA, et al. (2000): The prognostic value of anti-cyclic citrullinated peptide antibody in patients with recent onset rheumatoid arthritis. Arthritis Rheum 43: 1831-1835.

36. Kamradt T, Schubert D (2005): The role and clinical implications ofG6Pl in experimental models of rheumatoid arthritis. Arthritis Res Ther 7: 20-28.

37. Agrawal S, Misra R, Aggarwal A (2012): Autoantibodies in rheumatoid arthritis: association with severity of disease in established RA. Clin Rheumatol 26: 201-204.

38. Glasnovic M, Bosnjak I, Vcev A, et al. (2007): Anti-citrullinated antibodies, radiological joint damages and their correlations with disease activity score (DAS28). Coil Antropol 31: 345-348.

39. Viatte S, Plant D, Bowes J, et al. (2012): Genetic markers of rheumatoid arthritis susceptibility in anti-citrullinated peptide antibody negative patients. Ann Rheum Dis 71: 1984-1990.

40. Graham RR, Cotsapas C, Davies L, et al. (2008): Genetic variants near TNFAIP3 on 6q23 are associated with systemic lupus erythematosus. Nat Genet 40: 1059-1061.

41. Musone SL, Taylor KE, Lu TT, et al. (2008): Multiple polymorphisms in the TNFAIP3 region are independently associated with systemic lupus erythematosus. Nat Genet 40: 1062-1064.

42. Han JW, Zheng HF, Cui Y, et al. (2009): Genome-wide association study in a Chinese Han population identifies nine new susceptibility loci for systemic lupus erythematosus. Nat Genet 41: 1234-1237.

43. Cai LQ, Wang ZX, Lu WS, et al. (2010): A single-nucleotide polymorphism of the TNFAIP3 gene is associated with systemic lupus erythematosus in Chinese Han population. Mol Biol Rep 37: 389-394.

44. Zhong H, Li XL, Li M, et al. (2011): Replicated associations of TNFAIP3, TNIP1 and ETS1 with systemic lupus erythematosus in a southwestern Chinese population. Arthritis Res Ther 13: R186.

45. Kawasaki A, Ito I, Ito S, et al. (2010): Association of TNFAIP3 polymorphism with susceptibility to systemic lupus erythematosus in a Japanese population. J Biomed Biotechnol 2010: 207578.

46. Lodolce JP, Kolodziej LE, Rhee L, et al. (2010): African-derived genetic polymorphisms in TNFAIP3 mediate risk for autoimmunity. J Immunol 184: 7001-7009.

47. Palomino-Morales RJ, Rojas-Villarraga A, Gonzalez CI, et al. (2008): STAT4 but not TRAF1/C5 variants influence the risk of developing rheumatoid arthritis and systemic lupus erythematosus in Colombians. Genes Immun 9: 379-382.

48. Zervou MI, Vazgiourakis VM, Yilmaz N, et al. (2011): TRAF1/C5, eNOS, C1q, but not STAT4 and PTPN22 gene polymorphisms are associated with genetic susceptibility to systemic lupus erythematosus in Turkey. Hum Immunol 72: 1210-1213. 